\title{
The Shocking Truth: The Small Contribution to Hydrogen Reionization From Gravitational Infall
}

\section{Citation}

Wyithe, J. Stuart B., Jeremy Mould, and Abraham Loeb. 2011. "THE SHOCKING TRUTH: THE SMALL CONTRIBUTION TO HYDROGEN REIONIZATION FROM GRAVITATIONAL INFALL." The Astrophysical Journal 743 (2): 173. https://doi.org/10.1088/0004-637x/743/2/173.

\section{Permanent link}

http://nrs.harvard.edu/urn-3:HUL.InstRepos:41393171

\section{Terms of Use}

This article was downloaded from Harvard University's DASH repository, and is made available under the terms and conditions applicable to Other Posted Material, as set forth at http:// nrs.harvard.edu/urn-3:HUL.InstRepos:dash.current.terms-of-use\#LAA

\section{Share Your Story}

The Harvard community has made this article openly available.

Please share how this access benefits you. Submit a story.

Accessibility 


\title{
THE SHOCKING TRUTH: THE SMALL CONTRIBUTION TO HYDROGEN REIONIZATION FROM GRAVITATIONAL INFALL
}

\author{
J. Stuart B. Wyithe ${ }^{1,2}$, Jeremy Mould ${ }^{2,3}$, And Abraham LoeB ${ }^{4}$ \\ ${ }^{1}$ School of Physics, University of Melbourne, Parkville, Victoria 3010, Australia \\ ${ }^{2}$ ARC Centre of Excellence for All-sky Astrophysics (CAASTRO) \\ ${ }^{3}$ Center for Astrophysics and Super Computing, Swinburne University, Hawthorn, Victoria 3122, Australia \\ ${ }^{4}$ Astronomy Department, Harvard University, 60 Garden Street, Cambridge, MA 02138, USA \\ Received 2011 August 29; accepted 2011 October 19; published 2011 December 2
}

\begin{abstract}
It is commonly thought that stars are responsible for reionizing the universe. However, deep searches for star-forming galaxies during the epoch of reionization have not yet found sufficient galaxies to provide the necessary ionizing flux. Fast accretion shocks associated with gravitational infall of baryons during the formation of galaxies have recently been proposed as an alternative method of generating the required ionizing photons. Here we assess the contribution to hydrogen reionization from shocked gas associated with gravitational infall. We find that shocks can ionize no more than a few percent of the cosmic hydrogen by $z \sim 6$. However, the small fraction of ionizing radiation produced by fast accretion shocks would be significantly more biased than that associated with stars, leading to a modification of the luminosity weighted source clustering by $\sim 10 \%$. This modification of the bias may be measurable with future precision experiments utilizing the redshifted $21 \mathrm{~cm}$ line to study the distribution of hydrogen during the reionization era.
\end{abstract}

Key words: cosmology: theory - dark ages, reionization, first stars - diffuse radiation

\section{INTRODUCTION}

Star-bursting galaxies and quasars have been the leading candidates for the sources of the UV radiation required to reionize the hydrogen gas in the intergalactic medium (IGM; Loeb 2010). The quasar population is observed to decline quickly at $z \gtrsim 2.5$ (e.g., Fan et al. 2002) and so it is believed that galaxies contributed the bulk of UV photons that drove reionization (Madau et al. 1999; Srbinovsky \& Wyithe 2007; Bolton \& Haehnelt 2007). The observed number counts of highredshift galaxy candidates (Bouwens et al. 2011; Yan et al. 2010) discovered with the Hubble Ultra Deep Field (HUDF) have been used to build up a statistical description of starforming activity at redshift $z \gtrsim 7$. At $z \sim 7,8.6$, and 10.6, the flux limits correspond to absolute magnitudes $M_{\lim }=-18.0$, -18.3 , and $-18.6 \mathrm{mag}$, respectively. While impressively faint, these observations do not reach the levels corresponding to the faintest galaxies thought to exist at these early epochs (e.g., Barkana \& Loeb 2000), and the observed stars in the HUDF are insufficient to reionize the universe.

Trenti et al. (2010) have constructed an empirical model for the luminosity function and find that the observed population could have reionized the universe if it extends to luminosities fainter than observed. In addition to galaxies, the discovery of high-redshift gamma ray bursts (Salvaterra et al. 2009; Tanvir et al. 2009) can be used to probe the star formation rate up to $z \sim 8$ (Kistler et al. 2009), based on which Wyithe et al. (2010) found sufficient star formation to achieve reionization by $z \sim 6$.

Thus, there is evidence that stars are capable of reionizing the universe. However, until the sources are identified directly, it is prudent to study alternatives. One such alternative is provided by cooling radiation associated with shock-heated gas, which can process gravitational energy associated with structure formation into an ionizing radiation background. For example, Furlanetto \& Loeb (2004) have considered the effect of large-scale structure shocks on the IGM during reionization, finding that shocks heat the early IGM and produce a radiation background that effects molecular hydrogen formation and the spin temperature of neutral hydrogen. In a complementary study, Miniati et al. (2004) calculated the UV background from cooling radiation associated with virialized gas that is shock heated during halo formation. They evaluated the ionizing rate in the optically thin IGM at $z<6$, and determined that the ionizing background at the hydrogen ionization edge produced following virialization shocks was likely to be much smaller than for stars, unless supernova feedback could efficiently reheat the galactic gas (in which case the galaxies would likely produce a significant contribution to the ionizing flux from star light). Miniati et al. (2004) concluded that the hard spectrum produced may doubly reionize helium by $z \sim 6$, and also described the resulting effects on the thermal history of the IGM.

Recently, Dopita et al. (2011) presented high-resolution simulations of the shocks associated with the supersonic gravitational infall of gas onto a nascent galactic disk that arises when the cooling rate is much shorter than the free-fall time. They argued that this provided a source of photons that could augment the ionizing flux from galaxies, and indeed might dominate the reionization of the universe. If true this finding would have significant implications for studies of reionization. In particular, the direct link between star formation and the ionization structure of the IGM would be removed, so that future redshifted $21 \mathrm{~cm}$ experiments would not provide a fruitful route to study the first stars.

In this paper we assess the contribution to hydrogen reionization from ionizing photons produced in fast radiative accretion shocks. We note that virialization (Miniati et al. 2004) and fast accretion shocks (Dopita et al. 2011) should lead to similar ionizing luminosities since the cooling radiation following the virialization shock contains energy comparable to the gravitational potential energy available to drive the fast accretion shock. In our numerical examples, we adopt the standard set of cosmological parameters (Komatsu et al. 2011), with values of 
$\Omega_{\mathrm{b}}=0.04, \Omega_{\mathrm{m}}=0.24$, and $\Omega_{\Lambda}=0.76$ for the matter, baryon, and dark energy fractional density, respectively, $h=0.73$, for the dimensionless Hubble constant, and $\sigma_{8}=0.82$.

\section{IONIZING PHOTONS}

We begin by computing the number of ionizations per baryon processed through shocks. We also discuss the ionization history due to stars for comparison.

\subsection{Ionizing Photons Based on Collapsed Fraction}

Dopita et al. (2011) presented the results of a series of fast shock models for nearly pristine infalling gas, including the resulting spectrum, varying the shock velocity between 100 and $500 \mathrm{~km} \mathrm{~s}^{-1}$. They assume the shock to be running into a medium which is in collisional ionization equilibrium at $3 \times 10^{4} \mathrm{~K}$. From the resulting spectra, Dopita et al. (2011) presented a fitting formula for the number of ionizing photons that enter the IGM per baryon processed through a shock, which in our terminology is

$$
\begin{aligned}
& N_{\gamma}=0.2\left(\frac{v}{100 \mathrm{~km} \mathrm{~s}^{-1}}\right)^{2} \text { for } 100<v<280 \mathrm{~km} \mathrm{~s}^{-1} \\
& N_{\gamma}=10\left(\frac{v}{400 \mathrm{~km} \mathrm{~s}^{-1}}\right)^{5} \text { for } 280<v<400 \mathrm{~km} \mathrm{~s}^{-1} \\
& N_{\gamma}=10\left(\frac{v}{400 \mathrm{~km} \mathrm{~s}^{-1}}\right)^{2} \text { for } v>400 \mathrm{~km} \mathrm{~s}^{-1},
\end{aligned}
$$

where $v$ is the velocity of the shocked gas. Dopita et al. (2011) argue that $v=\sqrt{2} v_{\text {vir }}$ where $v_{\text {vir }}$ is the virial velocity of the halo, and we utilize this throughout the current work. The virial velocity is estimated from the halo mass using

$$
v_{\text {vir }}\left(M_{\text {halo }}, z\right)=23.4\left(\frac{M_{\text {halo }}}{10^{8} M_{\odot} h^{-1}}\right)^{\frac{1}{3}}[\zeta(z)]^{\frac{1}{6}}\left(\frac{1+z}{10}\right)^{\frac{1}{2}}
$$

where $\zeta(z)$ is close to unity and defined as $\zeta \equiv$ $\left[\left(\Omega_{m} / \Omega_{m}^{z}\right)\left(\Delta_{c} / 18 \pi^{2}\right)\right], \Omega_{m}^{z} \equiv\left[1+\left(\Omega_{\Lambda} / \Omega_{m}\right)(1+z)^{-3}\right]^{-1}, \Delta_{c}=$ $18 \pi^{2}+82 d-39 d^{2}$, and $d=\Omega_{m}^{z}-1$ (see Equations (22)- (25) in Barkana \& Loeb 2001 for more details).

Under the assumption that gas shocks once when a halo forms, the number of photons produced per hydrogen atom in the universe can be estimated from the Press \& Schechter (1974) mass function $d n / d M$ (with modifications due to Sheth \& Tormen 1999),

$$
\frac{n_{\gamma}}{n_{\mathrm{H}}}=\frac{1}{0.76 \rho_{\mathrm{b}}} \int_{M_{\text {halo }}}^{\infty} d M N_{\gamma} M_{\mathrm{b}} \frac{d n}{d M},
$$

where $M_{\mathrm{b}}=\left(\Omega_{\mathrm{b}} / \Omega_{\mathrm{m}}\right) M$ and $\rho_{\mathrm{b}}=\left(\Omega_{\mathrm{b}} / \Omega_{\mathrm{m}}\right) \rho_{\mathrm{m}}$ are the baryonic mass inside a dark matter halo and baryonic mass density in the IGM, respectively. Results are shown as a function of redshift in the upper left panel of Figure 1, assuming minimum halo masses corresponding to $v_{\text {vir }}=10 \mathrm{~km} \mathrm{~s}^{-1}$ (the cooling threshold for hydrogen), and $v_{\text {vir }}=30 \mathrm{~km} \mathrm{~s}^{-1}$ (the Jeans threshold in an ionized IGM; Dijkstra et al. 2004). The results are independent of this choice owing to the dominance of massive halos in producing radiation from fast accretion shocks (Dopita et al. 2011 ). We find that only a few percent of the IGM is reionized by $z \sim 6$, and $<1 \%$ at $z \sim 8$, indicating that there are 1-2 orders of magnitude too few ionizing photons produced in fast accretion shocks to reionize the universe.
We note that virialization shocks do not exist in low-mass halos due to the presence of cold flows. Given the halo mass dependent fraction of cold flow accretion $f_{\text {cold }}$ where no shock is produced (Kereš et al. 2009; Faucher-Giguère et al. 2010), one should exclude cold-mode accretion material from the calculation of ionizing luminosity associated with virialization shocks (Miniati et al. 2004). This would reduce the predictions in Miniati et al. (2004) by a factor of $\left(1-f_{\text {cold }}\right)$, where $f_{\text {cold }}$ is weighted over the mass and number of halos. However, Dopita et al. (2011) argue that with sufficient resolution the cold flow material is found to shock at the intersection with the nascent galactic disk. As a result no correction for cold flow accretion should be applied to estimates of fast accretion shock produced ionizing photons in Equation (3) or later in this paper.

\subsection{Ionizing Photons from Stars}

We compare the above result to the number of ionizations obtained for stars (lower right panel of Figure 1). Here we utilize Equation (3) but assume $N_{\gamma}=4000\left(f_{\star} f_{\text {esc }}\right)=15$, appropriate for a Salpeter IMF with a fiducial value of $f_{\star} f_{\text {esc }} \sim 0.004$, where $f_{\star}$ and $f_{\text {esc }}$ are the star formation efficiency and escape fraction of ionizing photons, respectively. We again show minimum halo masses corresponding to $v_{\mathrm{vir}}=10 \mathrm{~km} \mathrm{~s}^{-1}$ and $v_{\mathrm{vir}}=30 \mathrm{~km} \mathrm{~s}^{-1}$, leading to significant variation in the total number of ionizing photons produced. In difference to shock produced photons, these fiducial stellar populations are easily able to reionize the universe by $z \sim 6$ (as is well known). This result is plausible since the nuclear efficiency of stars is larger by many orders of magnitude than the efficiency of converting rest mass to radiation by a shock, $\sim(v / c)^{2} \lesssim 10^{-6}$, for the shock speeds of interest $\left(v \lesssim 300 \mathrm{~km} \mathrm{~s}^{-1}\right)$.

\subsection{Ionizing Photons Based on Merger Rates}

The calculation in Section 2.1 utilizes each baryon only once, whereas, unlike the case for stars, a baryon may be processed through shocks several times during the hierarchical formation of a galaxy. We therefore recalculate the contribution to reionization from fast accretion shocks based on the merger rate of halos. Specifically, when a halo of mass $M_{2}<M_{1}$ merges with a halo of mass $M_{1}$, we assume that the baryons contained within halo 1 are shocked at $v=\sqrt{2} v_{\text {vir }}\left(M_{1}+M_{2}\right)$. We assume that the star formation efficiency is negligible so that all baryons are available to shock during each merger. The resulting expression for the rate of production of all baryons in galaxies is

$$
\begin{aligned}
\frac{d}{d z}\left(\frac{n_{\gamma}}{n_{\mathrm{H}}}\right)= & \frac{1}{0.76 \rho_{\mathrm{b}}} \int_{M_{\text {halo }}}^{\infty} d M_{1} \frac{d n}{d M_{1}} \int_{M_{\text {halo }}}^{M_{1}} d M_{2} N_{\gamma} \\
& \times\left.\left(M_{\mathrm{b}, 1}+M_{\mathrm{b}, 2}\right) \frac{d^{2} N}{d z d M_{2}}\right|_{M_{1}},
\end{aligned}
$$

yielding

$$
\frac{n_{\gamma}}{n_{\mathrm{H}}}=\int_{\infty}^{z} d z^{\prime} \frac{d}{d z}\left(\frac{n_{\gamma}}{n_{\mathrm{H}}}\right) .
$$

Here, $\left.\frac{d^{2} N}{d z d M_{2}}\right|_{M_{1}}$ is the number of mergers of a halo with mass between $M_{2}$ and $M_{2}+d M_{2}$ that merge with a halo of mass $M_{1}$ in a redshift interval $d z$.

The assumption that baryons shock during each merger is optimistic. While mergers will certainly increase the virial energy per baryon, it is not clear how the baryons would be 

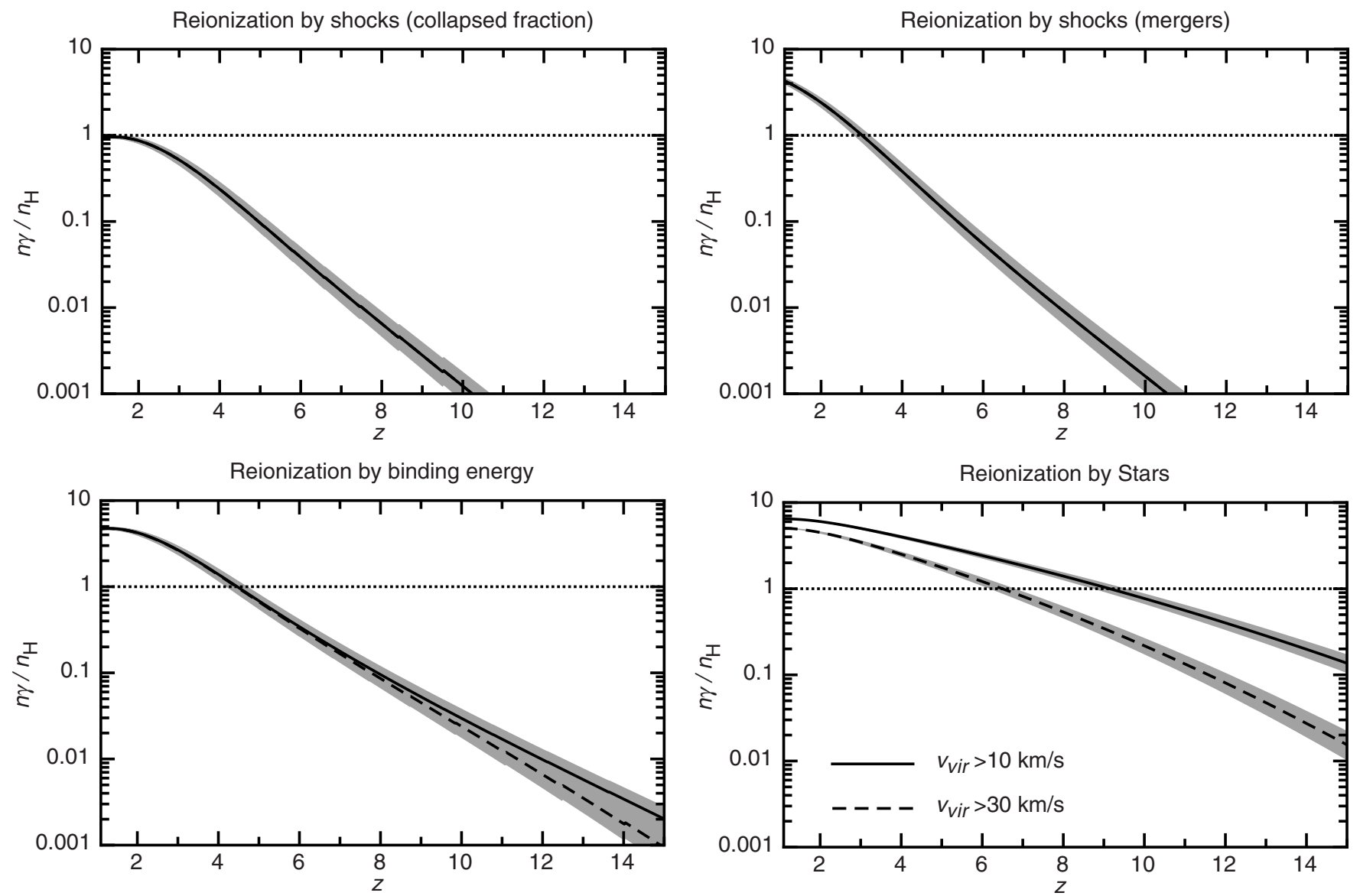

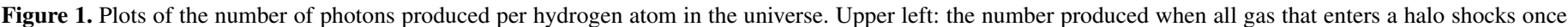

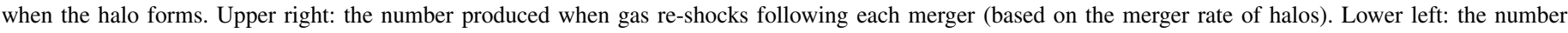

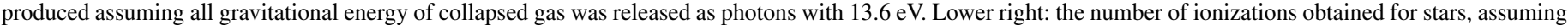

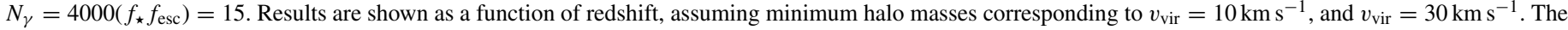
gray strips represent uncertainty in $\sigma_{8}$.

recycled through virialization shocks on multiple occasions if they cool efficiently to produce the ionizing radiation. If the baryons cool from the halo into a disk, they will not be heated to the virial temperature of the halo (unless they have been expelled through some feedback process) and so should not participate in the halo shock of the next merger. Thus, our results from Equation (4) represent the maximum ionization flux possible from the fast accretion shock mechanism summarized in Equation (1).

Results are shown as a function of redshift in the upper right panel of Figure 1, assuming minimum halo masses corresponding to $v_{\text {vir }}=10 \mathrm{~km} \mathrm{~s}^{-1}$ and $v_{\text {vir }}=30 \mathrm{~km} \mathrm{~s}^{-1}$ (again with no discernible difference owing to the dominance of massive halos). We find that the re-processing of baryons through shocks in multiple mergers increases the ionizing photon output by a factor of a few relative to the collapsed fraction calculation. However, we still find that only $\sim 5 \%$ of the IGM is reionized by $z \sim 6$, and $\sim 1 \%$ at $z \sim 8$, indicating that shocks provide insufficient ionizing luminosity to reionize the IGM.

The difference between our findings and the results presented in Dopita et al. (2011) originates partly from the adoption of a large value of $\sigma_{8}=0.9$ in that work, and partly from an error in the calculation method (L. Krauss 2011, private communication). Dopita et al. (2011) calculate the accretion rate and corresponding ionizing luminosity as a function of halo mass (see their Figure 2). They then integrate over the mass function and redshift. This procedure effectively sets the rate at which gas in the halo doubles to be equal to the inverse of dynamical time at the virial radius (which is shorter by an order of magnitude than the Hubble time), and so does not account for the duty cycle of the shocks (which should be only $\sim 0.1$ ). As a result, the calculation in Dopita et al. (2011) accretes an order of magnitude more gas than available per halo.

\subsection{A Maximum of Ionizing Photons Based on Binding Energy}

In the previous subsections we have estimated the number of ionizing photons available per hydrogen in the IGM. We next estimate the maximum number of photons that equal the binding energy of all baryons in the halo. This should provide an upper limit to the number of ionizing photons produced by shocks (in the absence of significant feedback; Miniati et al. 2004), and hence an upper limit on the contribution of shocks to reionization. For this calculation, we again appeal to Equation (3), setting $N_{\gamma}$ to be

$$
N_{\gamma}=\frac{\frac{1}{2} M_{\mathrm{b}} v_{\mathrm{vir}}^{2}}{13.6 \mathrm{eV}} .
$$

Results are shown in the lower left panel of Figure 1 assuming minimum halo masses corresponding to $v_{\mathrm{vir}}=10 \mathrm{~km} \mathrm{~s}^{-1}$ and 
$v_{\text {vir }}=30 \mathrm{~km} \mathrm{~s}^{-1}$. We find that the total gravitational energy available for ionization of hydrogen corresponds to less than 1 ionizing photon per 3 hydrogens by $z \sim 6$ and less than 1 ionizing photon per 10 hydrogens by $z \sim 8$.

\section{REIONIZATION HISTORIES}

Next we use the estimate of flux based on our merger calculation of ionizing radiation from shocks as the source term in a calculation of the reionization history. Miralda-Escudé et al. (2000) presented a model which allows the calculation of an effective recombination rate in an inhomogeneous universe by assuming a maximum overdensity $\left(\Delta_{\mathrm{c}}\right)$ penetrated by ionizing photons within $\mathrm{H}$ II regions. The model assumes that reionization progresses rapidly through islands of lower density prior to the overlap of individual cosmological ionized regions. Following the overlap epoch, the remaining regions of high density are gradually ionized. It is therefore hypothesized that at any time within reionized regions of the IGM, regions with gas below some critical overdensity $\Delta_{\mathrm{i}} \equiv \rho_{i} /\langle\rho\rangle$ are highly ionized while regions of higher density are not. The fraction of mass in regions with overdensity below $\Delta_{\mathrm{i}}$, is found from the integral

$$
F_{\mathrm{m}}\left(\Delta_{\mathrm{i}}\right)=\int_{0}^{\Delta_{\mathrm{i}}} d \Delta P_{\mathrm{V}}(\Delta) \Delta
$$

where $P_{\mathrm{V}}(\Delta)$ is the volume weighted probability distribution for $\Delta$. Miralda-Escudé et al. (2000) quote a fitting function that provides a good fit to the volume weighted probability distribution for the baryon density in cosmological hydrodynamical simulations. Wyithe \& Loeb (2003) employed this prescription within a semi-analytic model of reionization, as summarized below.

The quantity $Q$ is defined to be the volume filling factor within which all matter at densities below $\Delta_{\mathrm{i}}$ has been ionized. The reionization history is quantified by the evolution of $Q$ that evolves according to the rate equation

$$
\begin{aligned}
\frac{d Q}{d z}= & \frac{1}{n_{0} F_{\mathrm{m}}\left(\Delta_{\mathrm{i}}\right)}\left(\frac{d n_{\gamma, 10}}{d z}(1-Q)+\frac{d n_{\gamma, 30}}{d z} Q\right) \\
& -\left[\alpha_{\mathrm{B}}(1+z)^{3} R\left(\Delta_{\mathrm{i}}\right) n_{0} \frac{d t}{d z}+\frac{d F_{\mathrm{m}}\left(\Delta_{\mathrm{i}}\right)}{d z}\right] \frac{Q}{F_{\mathrm{m}}\left(\Delta_{\mathrm{i}}\right)},
\end{aligned}
$$

where $\alpha_{\mathrm{B}}$ is the case $\mathrm{B}$ recombination coefficient, $n_{0}$ is the comoving density of hydrogen in the mean IGM, and $R\left(\Delta_{\mathrm{i}}\right)$ is the effective clumping factor of the IGM. The corresponding massaveraged ionization fraction is $Q_{\mathrm{m}}=Q F_{\mathrm{m}}$. The evolution is driven by the rate of emission of ionizing photons per comoving volume $d n_{\gamma} / d z$, which is the weighted sum of the ionization rates in neutral and ionized regions (signified in Equation (8) by the subscripts 10 and 30, respectively).

Within this formalism, the epoch of overlap is precisely defined as the time when $Q$ reaches unity. Prior to the overlap epoch we must solve for both $Q$ and $F_{\mathrm{m}}$ (or equivalently $\Delta_{\mathrm{i}}$ ). The relative growth of these depends on the luminosity function and spatial distribution of the sources. In this regime we assume $\Delta_{\mathrm{i}}$ to be constant with a critical value $\Delta_{\mathrm{c}}$.

Following overlap we may describe the post-overlap evolution of the IGM by computing the evolution of the ionized mass fraction according to the equation

$$
\frac{d F_{\mathrm{m}}\left(\Delta_{\mathrm{i}}\right)}{d z}=\frac{1}{n_{0}} \frac{d n_{\gamma, 30}}{d z}-\alpha_{\mathrm{B}}(1+z)^{3} R\left(\Delta_{\mathrm{i}}\right) n_{0} \frac{d t}{d z} .
$$

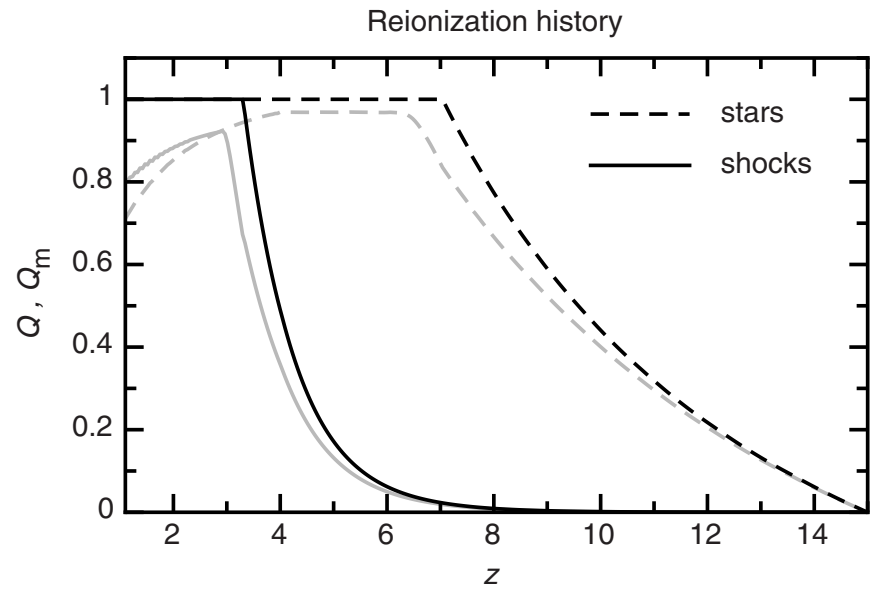

Figure 2. Plots of the reionization history of the IGM and the subsequent postoverlap evolution due to ionizing sources from merger-induced shocks (solid lines), and stars with $N_{\gamma}=15$ (dashed lines). The case shown corresponds to a value for the critical overdensity prior to the overlap epoch of $\Delta_{\mathrm{c}}=5$, and both the volume-averaged (thick lines) and mass-averaged (thin lines) ionization fractions are shown.

Note that this follows directly from Equation (8) with $Q=1$, and that in this post overlap regime the value of $\Delta_{\mathrm{i}}$ is the dependent variable describing the ionization state of the IGM (whereas prior to overlap $\Delta_{\mathrm{i}}=\Delta_{\mathrm{c}}$ ). Equation (9) is integrated to obtain $F_{\mathrm{M}}$ (or equivalently $\Delta_{\mathrm{i}}$ ) as a function of redshift.

Figure 2 shows the resulting model for the reionization of the IGM and the subsequent post-overlap evolution due to ionizing sources from fast accretion shocks (solid lines). Here we have used an ionizing photon rate based on Equation (4). The case shown corresponds to a value for the critical overdensity prior to the overlap epoch of $\Delta_{c}=5$, and both the volumeaveraged (dark lines) and mass-averaged (gray lines) ionization fractions are shown. We find that shocks can reionize less than $10 \%$ of the IGM (by volume or mass) prior to $z \sim 6$, and cannot complete reionization until $z \sim 3$. For comparison we compute the reionization history for stars (dashed lines), where the ionizing photon production rate is based on $d\left(n_{\gamma} / n_{\mathrm{H}}\right) / d z$, with $n_{\gamma} / n_{\mathrm{H}}$ based on Equation (3) with $N_{\gamma}=15$. In this model, stars complete reionization by $z \sim 8$, at which time the relative contributions from stars and fast accretion shocks differ by a factor in excess of 100 .

\section{IMPLICATIONS FOR THE BIAS OF IONIZING SOURCES AND $21 \mathrm{~cm}$ STUDIES}

In this paper we have demonstrated that recent estimates of the ionizing luminosity from fast accretion shocks associated with galaxy formation are not sufficient to drive reionization. However, the ionizing photons produced by shocks are dominated by massive halos (Miniati et al. 2004; Dopita et al. 2011). This is in contrast to the ionizing radiation from stars, which is both predicted and observed to be dominated by lowmass galaxies. As a result, the ionizing radiation produced in shocks is significantly more biased relative to the underlying large-scale density of the IGM than are ionizing photons produced in galaxies. It is easy to see the physics of the dominance of massive halos by noting that the collapse energy available in Equation (6) is proportional to $v_{\mathrm{vir}}^{6}$ (or $M_{\mathrm{halo}}^{2}$ ), whereas the stellar mass (assuming a constant mass-to-light ratio) is proportional to $v_{\text {vir }}^{3}\left(\right.$ or $\left.M_{\text {halo }}\right)$.

The ionization structure of the IGM, particularly the scale of $\mathrm{HII}$ regions produced, is a sensitive function of the bias 

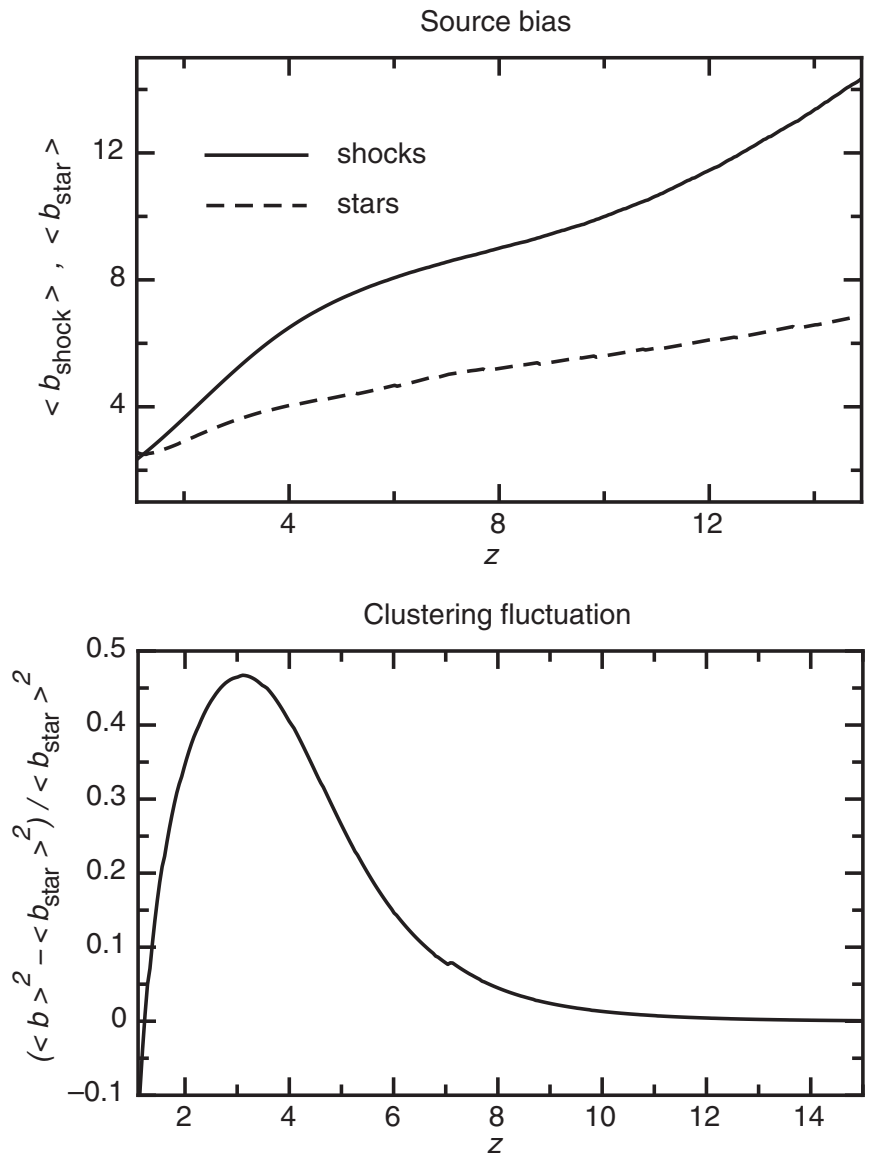

Figure 3. Plots of the luminosity weighted galaxy bias. Upper panel: the bias is plotted as a function of redshift due to ionizing sources from merger-induced shocks (solid lines), and stars with $N_{\gamma}=15$ (dashed lines). Lower panel: the fractional change in observed clustering of ionizing radiation when compared with the stars-only history.

of ionizing sources (McQuinn et al. 2007). It is this relation between the bias of ionizing sources and the resulting ionization structure during reionization that motivates redshifted $21 \mathrm{~cm}$ experiments with the ultimate aim of connecting galaxy properties to the power spectrum of $21 \mathrm{~cm}$ fluctuations (Barkana 2009). Here we quantify the effect of fast accretion shocks on the bias of ionizing sources. The halo bias $b$ for a halo mass $M$ at redshift $z$ may be approximated using the Press \& Schechter (1974) formalism, modified to include non-spherical collapse (Sheth et al. 2001). The power spectrum of the space distribution of sources is proportional to $b$ squared. The luminosity weighted bias of ionizing radiation produced by shocks arising in mergers can be evaluated using the expression

$$
\begin{aligned}
\left\langle b_{\text {shock }}\right\rangle= & {\left[\frac{d}{d z}\left(\frac{n_{\gamma}}{n_{\mathrm{H}}}\right)\right]^{-1} \frac{1}{0.76 \rho_{\mathrm{b}}} \int_{M_{\text {halo }}}^{\infty} d M_{1} \frac{d n}{d M_{1}} } \\
& \times \int_{M_{\text {halo }}}^{M_{1}} d M_{2} N_{\gamma} \times\left.\left(M_{\mathrm{b}, 1}+M_{\mathrm{b}, 2}\right) \frac{d^{2} N}{d z d M_{2}}\right|_{M_{1}} b,
\end{aligned}
$$

where the bias $b$ is evaluated at a mass $M_{1}+M_{2}$. The resulting bias is plotted as a function of redshift in the upper panel of Figure 3. Prior to reionization, fast accretion shock powered ionizing sources have a luminosity weighted bias of $\left\langle b_{\text {shock }}\right\rangle \sim 10$.
For comparison, we calculate the luminosity weighted bias for stellar sources $\left(\left\langle b_{\text {star }}\right\rangle\right)$ based on the derivative of Equation (3), and in analogy with Equation (4). The result is also plotted in the upper panel of Figure 3. Prior to reionization, stellar ionizing sources have a smaller luminosity weighted bias of $\left\langle b_{\text {star }}\right\rangle \sim 4$. Finally, we evaluate the luminosity weighted bias $\langle b\rangle$ obtained when fast accretion shock powered ionization sources are added to the stellar sources needed for reionization. We then calculate the fractional change in observed clustering of ionizing radiation relative to the stars-only reionization history $\left[\left(\langle b\rangle^{2}-\left\langle b_{\text {star }}\right\rangle^{2}\right) /\left\langle b_{\text {star }}\right\rangle^{2}\right]$. This fractional change is plotted in the lower panel of Figure 3, which shows that the power spectrum of ionizing sources is increased by $\sim 10 \%$ owing to ionizing radiation produced in shocks. This change in the clustering of ionizing sources will lead to comparable changes in the amplitude of redshifted $21 \mathrm{~cm}$ fluctuations (Wyithe \& Morales 2007) that will be detectable by planned low-frequency radio telescopes (Wyithe et al. 2009). For example, as illustrated in the analysis of Lidz et al. (2008) the planned Murchison Widefield Array would make a measurement of the amplitude of the $21 \mathrm{~cm}$ power spectrum with better than $\sim 10 \%$ precision over a $24 \mathrm{MHz}$ bandpass during $1000 \mathrm{hr}$ of integration.

The effects of ionization by shocks on the amplitude of the $21 \mathrm{~cm}$ power spectrum are likely to be degenerate with those of increased bias in the stellar sources. However, shocks are likely to introduce additional effects on galaxy formation through temperature increases in the IGM, and through the production of high-energy photons with long mean free paths that ionize the IGM more uniformly than do the UV photons produced in stars. Our analysis indicates that shocks will need to be considered in detailed modeling of the $21 \mathrm{~cm}$ intensity power spectrum, in order to correctly interpret measurements from forthcoming low-frequency arrays.

The estimate of the relative increase in the clustering signal shown in Figure 3 is likely a lower limit. First, our reionization modeling assumes that the minimum mass for star formation is set by the virial temperature at which hydrogen can cool efficiently and form stars. However, in primordial gas molecular hydrogen cooling can lead to star formation in lower mass halos than can atomic hydrogen cooling, thus lowering the predicted bias of the stellar sources. Moreover in reionized regions our modeling assumes the minimum mass is set by the Jeans mass in an ionized IGM with an assumed virial velocity of $30 \mathrm{~km} \mathrm{~s}^{-1}$, while at high redshift the radiative feedback may not be efficient (Dijkstra et al. 2004). Thus, the bias of stellar sources may be lower than assumed in the calculation of Figure 3, and hence the relative increase in the bias from the contribution of shocks may be higher than the $10 \%$ value estimated here.

\section{CONCLUSION}

Based on recent high-resolution simulations (Dopita et al. 2011), we have quantified the contribution that gravitationally powered fast accretion shocks during galaxy formation can make to the reionization of hydrogen. We find that ionizing radiation from fast accretion shocks represents a negligible contribution to the overall reionization history of hydrogen, leaving the dominant contribution to be provided by stars. This result is in agreement with the estimates from Miniati et al. (2004) who found that the ionizing background at the hydrogen ionization edge produced following virialization shocks was likely to be more than an order of magnitude smaller than for stars. Our conclusion is also consistent with expectations 
based on observations of cosmic background radiation. The energy released by star formation at high redshift is stored in the cosmic infrared background with $v L_{v} \sim 10 \mathrm{nW} \mathrm{m}^{-2} \mathrm{Str}^{-1}$ (Hauser \& Dwek 2001). On the other hand, any energy surplus from gravitational shocks is stored in the cosmic soft X-ray background at $\sim 0.01 \mathrm{nW} \mathrm{m} \mathrm{Str}^{-1}$. This three orders of magnitude difference is suggestive of the relative efficiency of shocks and star formation in illuminating the universe at high redshift.

Despite their small contribution to hydrogen reionization, shocks may have observable consequences for studies of the reionization era. As discussed by Miniati et al. (2004), the harder spectrum associated with shocks will lead to a modification of the thermal history. In particular, the reionization of hydrogen by shocks would be accompanied by reionization of singly ionized helium, thus heating the IGM to levels above those observed at $z \sim 5$ Becker et al. (2011). While this likely rules out reionization by shocks, independently from the hydrogen ionization photon budget, heating by shocks may still have important consequences for star formation at high redshift (e.g., Dijkstra et al. 2004).

In addition, we find that because the small contribution from fast accretion shocks is produced in highly biased galaxies, their presence modifies the mean clustering bias of the combined ionizing radiation. This modification will likely lead to observable changes in the redshifted $21 \mathrm{~cm}$ fluctuations from neutral hydrogen during reionization, and so will need to be considered in analyses which aim to use precision measurements of $21 \mathrm{~cm}$ fluctuations to study the properties of very high redshift galaxies.

We thank James Bolton, Jonathan Bittner, and Lawrence Krauss for helpful discussions during this work. The Centre for All-sky Astrophysics is an Australian Research Council Centre of Excellence, funded by grant CE11E0090. A.L. was supported in part by NSF grant AST-0907890 and NASA grants NNX08AL43G and NNA09DB30A.

\section{REFERENCES}

Barkana, R. 2009, MNRAS, 397, 1454

Barkana, R., \& Loeb, A. 2000, ApJ, 539, 20

Barkana, R., \& Loeb, A. 2001, Phys. Rep., 349, 125

Becker, G. D., Bolton, J. S., Haehnelt, M. G., \& Sargent, W. L. W. 2011, MNRAS, 410, 1096

Bolton, J. S., \& Haehnelt, M. G. 2007, MNRAS, 382, 325

Bouwens, R. J., Illingworth, G. D., Oesch, P. A., et al. 2011, ApJ, 737, 90

Dijkstra, M., Haiman, Z., Rees, M. J., \& Weinberg, D. H. 2004, ApJ, 601, 666

Dopita, M. A., Krauss, L. M., Sutherland, R. S., Kobayashi, C., \& Lineweaver, C. H. 2011, Ap\&SS, 335, 345

Fan, X., Narayanan, V. K., Strauss, M. A., et al. 2002, AJ, 123, 1247

Faucher-Giguère, C.-A., Kereš, D., Dijkstra, M., Hernquist, L., \& Zaldarriaga, M. 2010, ApJ, 725, 633

Furlanetto, S. R., \& Loeb, A. 2004, ApJ, 611, 642

Hauser, M. G., \& Dwek, E. 2001, ARA\&A, 39, 249

Kereš, D., Katz, N., Fardal, M., Davé, R., \& Weinberg, D. H. 2009, MNRAS, 395,160

Kistler, M. D., Yüksel, H., Beacom, J. F., Hopkins, A. M., \& Wyithe, J. S. B. 2009, ApJ, 705, L104

Komatsu, E., Smith, K. M., Dunkley, J., et al. 2011, ApJS, 192, 18

Lidz, A., Zahn, O., McQuinn, M., Zaldarriaga, M., \& Hernquist, L. 2008, ApJ, 680,962

Loeb, A. 2010, How Did the First Stars and Galaxies Form? (Princeton, NJ: Princeton Univ. Press)

Madau, P., Haardt, F., \& Rees, M. J. 1999, ApJ, 514, 648

McQuinn, M., Lidz, A., Zahn, O., et al. 2007, MNRAS, 377, 1043

Miniati, F., Ferrara, A., White, S. D. M., \& Bianchi, S. 2004, MNRAS, 348, 964

Miralda-Escudé, J., Haehnelt, M., \& Rees, M. J. 2000, ApJ, 530, 1

Press, W. H., \& Schechter, P. 1974, ApJ, 187, 425

Salvaterra, R., Della Valle, M., Campana, S., et al. 2009, Nature, 461, 1258

Sheth, R. K., Mo, H. J., \& Tormen, G. 2001, MNRAS, 323, 1

Sheth, R. K., \& Tormen, G. 1999, MNRAS, 308, 119

Srbinovsky, J. A., \& Wyithe, J. S. B. 2007, MNRAS, 374, 627

Tanvir, N. R., Fox, D. B., Levan, A. J., et al. 2009, Nature, 461, 1254

Trenti, M., Stiavelli, M., Bouwens, R. J., et al. 2010, ApJ, 714, L202

Wyithe, J. S. B., Hopkins, A. M., Kistler, M. D., Yüksel, H., \& Beacom, J. F. 2010, MNRAS, 401, 2561

Wyithe, J. S. B., \& Loeb, A. 2003, ApJ, 586, 693

Wyithe, J. S. B., \& Morales, M. F. 2007, MNRAS, 379, 1647

Wyithe, J. S. B., Warszawski, L., Geil, P. M., \& Oh, S. P. 2009, MNRAS, 395, 311

Yan, H.-J., Windhorst, R. A., Hathi, N. P., et al. 2010, Res. Astron. Astrophys., 10, 867 